\title{
Article
}

\section{Fluctuating Epistemic Uncertainty in a Design Team as a Metacognitive Driver for Creative Cognitive Processes}

Christensen, Bo T. and Ball, Linden

Available at http://clok.uclan.ac.uk/20609/

Christensen, Bo T. and Ball, Linden ORCID: 0000-0002-5099-0124 (2017)

Fluctuating Epistemic Uncertainty in a Design Team as a Metacognitive Driver for Creative Cognitive Processes. CoDesign, 14 (2). pp. 133-152. ISSN 15710882

It is advisable to refer to the publisher's version if you intend to cite from the work. http://dx.doi.org/10.1080/15710882.2017.1402060

For more information about UCLan's research in this area go to http://www.uclan.ac.uk/researchgroups/ and search for <name of research Group>.

For information about Research generally at UCLan please go to http://www.uclan.ac.uk/research/

All outputs in CLoK are protected by Intellectual Property Rights law, including Copyright law. Copyright, IPR and Moral Rights for the works on this site are retained by the individual authors and/or other copyright owners. Terms and conditions for use of this material are defined in the policies page.

\section{CLoK}

Central Lancashire online Knowledge www.clok.uclan.ac.uk

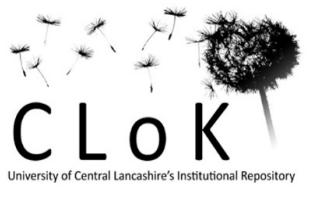




\section{Fluctuating Epistemic Uncertainty in a Design Team as a Metacognitive Driver for Creative Cognitive Processes}

Bo Thomas Christensen ${ }^{\mathrm{a}}$, Linden J. Ball ${ }^{\mathrm{b}}$

${ }^{a}$ Copenhagen Business School, Solbjerg Plads 3, 2000 Frederiksberg C, Denmark, phone: +45 38152123, bc.marktg@cbs.dk. Corresponding author.

${ }^{b}$ School of Psychology, Darwin Building, University of Central Lancashire, Preston PRI 2HE, UK, phone: (01772) 893421; LBall@uclan.ac.uk

WORDCOUNT: 7153 words not counting abstract and references (Approx. 1200 of the wordcount consist of two extensive qualitative examples of coding)

ACKNOWLEDGEMENTS: This work was supported by Innovation Fund Denmark under grant CIBIS 1311-00001B, 'Creativity in Blended Interaction Spaces'. 


\section{ABSTRACT}

Previous design research has demonstrated how epistemic uncertainty engenders localized, creative reasoning, including analogizing and mental simulation. We analyzed not just the short-term, localized effects of epistemic uncertainty on creative processing and information selection, but also its long-term impact on downstream creative processes. Our hypothesis was that heightened levels of uncertainty associated with a particular cognitive referent would engender: (1) immediate creative elaboration of that referent aimed at resolving uncertainty and determining information selection; and (2) subsequent attentive returns to that cognitive referent at later points in time, aimed at resolving lingering uncertainty and determining information selection. Findings: First - contrary to expectations - we observed that increased epistemic certainty (rather than increased epistemic uncertainty) in relation to cognitive referents triggered immediate, creative reasoning and information elaboration. Second, epistemic uncertainty was, as predicted, found to engender subsequent attentive returns to cognitive referents. Third, although epistemic uncertainty did not predict the selection of information, both immediate creative elaboration and subsequent attentive returns did predict information selection, with subsequent attentive returns being the stronger predictor. Our findings hold promise for identifying more global impacts of epistemic uncertainty on creative design cognition possibly mediated through the establishment of lasting associations with cognitive referents.

Keywords: epistemic uncertainty, design cognition, creativity, cross-cultural interpretation, metacognition 


\section{THEORETICAL FRAMEWORK}

It is essential that the design process incorporates knowledge of end-users through user-oriented approaches such as anthropological investigations, user-driven design and participatory design. However, understanding users, especially across cultural divides, is a daunting task, as many a failed design artifact illustrates. Although cross-cultural interpretation can be a source of design error and failure, we suggest that it can also act as a catalyst for creative design. That is, because cross-cultural interpretation is frequently uncertain, ambiguous, re-frameable, contextually shiftable and open to exploration, it embodies the essential qualities that provide design objects and preinventive structures with creative potential, as captured by dominant theories of design and creativity (e.g., Dorst \& Cross, 2001; Finke, Ward \& Smith, 1995; Schön \& Wiggins, 1992). Indeed, much design-reasoning research has convincingly demonstrated that effective designers are not only at ease with uncertainty but thrive in relation to the opportunities it affords (AlcaideMarzal, Diego-Más, Asensio-Cuesta, \& Piqueras-Fiszman, 2013; McDonnell, 2015; Schön, 1983). A few previous studies have addressed the importance of uncertainty in design, although not with a focus on cross-cultural interpretation. Beheshti (1993) discussed uncertainty as a key factor influencing design decisions, noting that it is important to minimize its influence so as to increase decision quality. We likewise see uncertainty as a pervasive aspect of design and view it positively since it provides valuable opportunities for creative ideation as part of the process of uncertainty reduction. Designers also view uncertainty as a positive element of their professional self-identity, as shown in Tracey and Hutchinson's (2016) qualitative study of designers who were prompted to reflect on their experiences and beliefs regarding uncertainty. D'souza and Dastmalchi (2017) discuss uncertainty in design jargon and slang usage, while Paletz, Sumer, and Miron-Spektor (2017) relate uncertainty to design team micro-conflicts. 
Our current analysis of made use of ethnographic design data stemming from the Design Thinking Research Symposium 11 (Christensen, Ball \& Halskov, 2017), and focused on the extent to which uncertainty arising specifically from cross-cultural interpretation elicits creative design reasoning both in the short-term (e.g., engendering localized analogizing and mental simulation) and in the longer-term (influencing downstream creative processes and decision-making). To address this issue we examined those parts of the dataset that involved the Scandinavian design team comprehending and analyzing a large set of lead-user generated post-it notes written in Chinese. Our overarching assumption was that uncertainties in the interpretation of these post-it notes (preinventive structures) would be likely to promote creative processes and subsequent returns to information, eventually predicting what information would be extracted by the team to be taken forward.

\subsection{Epistemic Uncertainty as a Metacognitive Trigger for Creative Analysis}

The concept of uncertainty that we draw upon for our analysis is that of 'epistemic uncertainty', which refers to a designer's experienced, subjective and fluctuating feelings of confidence in their knowledge and choices, as measured through phrases in the design dialogue. This epistemic uncertainty is differentiable from 'aleatory uncertainty', which is expressed in natural language via likelihood statements (Ülkümen, Fox, \& Malle, 2016). Heightened levels of epistemic uncertainty appear to act as a 'metacognitive cue' (Ackerman \& Thompson, 2014; Alter \& Oppenheimer, 2009; Alter, Oppenheimer, Epley, \& Eyre, 2007; Ball \& Stupple, 2016; Thompson, Prowse Turner \& Pennycook, 2011; Thompson et al., 2013), triggering more elaborate reasoning than might otherwise arise when people feel confident about ongoing processing. Similar ideas are noted by Stempfle and Badke-Schaub (2002) in a study of design-team thinking that coded for expressions of 
uncertainty. Their findings suggest that simpler design problems are associated with team selfefficacy and rapid and intuitive evaluative reasoning, whereas complex design problems may trigger a shift toward a structured process of effortful idea generation and analysis.

Epistemic uncertainty has previously been associated with creative analogizing (e.g., Dunbar, 1997; Houghton, 1998) and mental simulation (e.g., Nersessian, 2009). Indeed, spikes in expressed uncertainty reliably predict analogizing in engineering design (Ball \& Christensen, 2009) and scientific problem solving (Chan, Paletz \& Schunn, 2012), with these studies demonstrating that analogizing subsequently reduces uncertainty to baseline levels. Similarly, mental simulations during design have been found to be run in situations of elevated epistemic uncertainty (Ball \& Christensen, 2009; Ball, Onarheim, \& Christensen, 2010; Christensen \& Schunn, 2009) and function strategically to reduce uncertainty through the generation of approximate answers to design issues. In addition, strategic switches between depth-first and breadth-first design moves have been shown to be mediated by epistemic uncertainty (Ball, Onarheim, \& Christensen, 2010) and episodes of problem-solution co-evolution also take place under elevated levels of epistemic uncertainty (Wiltschnig, Christensen, \& Ball (2013), with solution attempts within these episodes being closely associated with uncertainty above baseline levels.

\subsection{Research Questions}

The underpinning assumption in these aforementioned studies is that a heightened level of epistemic uncertainty immediately sparks off a localized, creative episode (e.g., involving analogizing) aimed at reducing uncertainty. However, what has not been investigated is the degree to which increased epistemic uncertainty might also affect design across episodes that extend beyond the localized micro-situation in which it is experienced. No doubt the failure to address this 
research question reflects the methodological challenge of tracing uncertainty referents over time in naturally-occurring design dialogue. Answering this question requires shifting the unit of analysis from standard, sequential discourse segmentation (e.g., turn-taking in dialogue) to a focus on the qualities of the cognitive referents themselves, tracing their occurrence both locally (within microepisodes) and globally (across episodes).

In our analysis we sought to address head-on the question of whether the epistemic uncertainty initially associated with a cognitive referent predicts repeated referrals back to that referent. This might arise from designers utilizing information that is generated or encountered later in the design process in an attempt to address an earlier, epistemically-uncertain design issue that remains unresolved. A similar phenomenon is found in the classic literature on the function of memory in problem solving and concerns the 'Zeigarnik effect' (Zeigarnik, 1927), whereby people's memory for unsolved problems exceeds that for solved problems, indicating a special 'cognitive alertness' towards unanswered issues. This cognitive alertness might enable later, chance encounters with relevant stimuli to engender productive solution attempts (Christensen \& Schunn, 2005; Seifert et al., 1995; Yaniv \& Meyer, 1987) according to what has been termed the 'prepared mind hypothesis'. This idea is central to the 'opportunistic assimilation' theory of incubation effects, where incubation is the phenomenon whereby a period of time away from a problem and engaged in unrelated activities leads to enhanced solution likelihood on returning to the problem (Howard et al., 2008; Sio \& Ormerod, 2009; Gilhooly, in press).

In light of these past findings we propose that it is theoretically plausible that epistemic uncertainty will become associated with its cognitive referent (the object of the uncertainty) so as to influence the design situation at later stages that are temporally remote from the original occurrence of the uncertainty. In our current analysis, epistemic uncertainty was estimated based on the initial translation and elaboration of post-its by the design team. We then examined whether the team 
spent time immediately on local, creative elaboration on a post-it and on whether (and to what degree) the team turned its attention to the post-it at a later time.

\subsection{Hypotheses}

We hypothesized that elevated levels of epistemic uncertainty would predict local, creative processing $(X \rightarrow M 1$; Figure 1) as well as returns to the cognitive referent over time $(X \rightarrow M 2)$, as per the Zeigarnik effect. We also predicted that both the local, creative micro-episodes and the subsequent returns would predict which information was salient to the team and worth taking forward. In sum, we propose an overarching 'double mediation model' (Figure 1) in which epistemic uncertainty (X; independent variable) on initial encounters with individual post-its predicts: (1) the immediate occurrence of local, creative micro-episodes (M1; mediator) that mediate the formation of new post-its (information selection, Y; dependent variable); and (2) the occurrence of subsequent attentive returns across episodes (M2; mediator) that also lead to the formation of new post-its (information selection, Y).

Insert Figure 1 about here

Figure 1. The proposed double mediation model

Past research investigating epistemic uncertainty in design has focused on the triggering of creative processes. In the present analysis, however, it was also possible to trace the immediate and delayed outcomes of creative processes on the information selected to be taken forward. The focus was, therefore, specifically on whether the epistemic uncertainty in the initial encounter with the 
cognitive referent (i.e., a post-it or group of post-its) would affect both within-episode and betweenepisode creative cognition and information selection.

\section{METHODS}

We analyzed an extensive video-based dataset that was collected for the purpose of the Design Thinking Research Symposium 11 (Christensen, Ball \& Halskov, 2017), tracing a Scandinavian design team for 4 months, working for a European car manufacturer in designing, conducting, and analyzing co-creation sessions with Chinese lead-users. We applied 'in vivo' analysis (Christensen \& Ball, 2014; Dunbar, 1995) to the dataset, which involves studying expertise 'online' as it arises naturally. The in vivo methodology takes a particular stance on data analysis, with verbal data (including data from team discussions) being coded using a similar approach to that deployed when analyzing concurrent think-aloud protocols (Ericsson \& Simon, 1999).

\subsection{Video Selection and Protocol Coding}

We selected Videos 15-17 (Christensen \& Abildgaard, 2017) since these related to the design team translating into English the post-its that had been written by Chinese lead-users, with the designers sometimes elaborating on these post-its and generating new ideas. The team members subsequently noted down (on new post-its) selected information to take forward. The observed activity clearly involved more than straightforward translation between languages since it also included rich, inferential processes, with the designers mapping across information, making generalizations, deriving cultural meaning and extending information into design ideas. Moreover, for the designers, the process of deciphering what lead-users 'meant' was fraught with uncertainty. The dataset therefore provided a unique testbed for addressing our research questions regarding the impact of epistemic uncertainty on design cognition both within and across episodes. 
The selected videos had been recorded back-to-back over a single day, thus varying minimally in temporal factors that might have influenced the design process. In the videos, the Scandinavian design team had finalized co-creation workshops with Chinese lead-users, and then spent approximately 109 minutes going through the Chinese lead-user post-its, moderated by consultants capable of translating the information. The post-its thus supported design team cognition (Dove et al., 2017) Essentially, these sessions constituted iterations of Chinese post-it translations that resulted in some of the translated information being developed by the design team, with the information being selected and documented in English on separate posters to be taken forward.

\subsubsection{Coding cognitive referral segments and cognitive referral episodes}

The videos were transcribed and segmented by turn-taking of dialogue, resulting in 999 segments. To trace post-it usage and development, all post-its were numbered and categorized according to the poster and poster sub-section they were situated on and when they were added and moved. The data-segments were subsequently sub-divided by coding for post-it referral using gesture and dialogue. When a member of the design team referenced a post-it this was coded as a 'cognitive referral' in the associated dialogue segment, and whenever a segment contained mentions of more than one post-it referent, that segment was subdivided to ensure unique cognitive referents for each segment. This re-segmentation procedure resulted in 1158 segments.

Based on the coding of cognitive referral relating to post-its we then coded for 'cognitive referral episodes', which reflected clusters of segments pertaining to the same Chinese post-it or post-it cluster. This led to 89 episodes. Individual episode-segments contained translations of a post-it together with further elaborative comments aimed at trying to understand its meaning (e.g., by 
referencing Chinese cultural or contextual information). These cognitive referral episodes constituted our final unit of analysis.

\subsubsection{Coding epistemic uncertainty}

The coding for epistemic uncertainty followed the coding scheme used extensively in past research (e.g., Ball \& Christensen, 2009; Chan \& Schunn, 2012; Christensen \& Schunn, 2009; Trickett et al, 2005). It involved a syntactic approach whereby 'hedge words' are used to locate segments displaying uncertainty (e.g., 'probably', 'sort of', 'guess', 'maybe', 'possibly', 'don't know', '[don't] think', '[not] certain' and 'believe'). Segments containing these words were located and coded as 'uncertainty present' if it was clear from manual screening that the hedge words were not being stated as politeness markers or were otherwise not evidence of epistemic uncertainty. All instances of epistemic uncertainty were counted for each cognitive referral episode. Given the cognitive referral episodes contained multiple segments, the measure of epistemic uncertainty was a continuous one calculated by dividing the number of epistemically uncertain statements by the number of episode segments.

\subsubsection{Coding immediate creative elaboration}

Protocol segments that immediately followed a cognitive referral episode were coded for whether they revealed further generative and creative development of the episode content beyond what was derivable from the cognitive referent (e.g., analogizing, idea generation and old-new information synthesis). Segments were coded in a binary manner as 'immediate creative elaboration present' versus 'immediate creative elaboration absent'. 


\subsubsection{Coding subsequent attentive returns to the cognitive referent}

To measure subsequent attentive returns to a cognitive referent we tabulated the number of segments referring back to each cognitive referent. We then conducted a mean-split to divide the episodes into ones with many subsequent attentive returns versus few subsequent attentive returns.

\subsubsection{Coding information selection}

Based on the cognitive referral code, all new post-it generation was related to the episodes, allowing for an analysis of which Chinese post-its were linked to the resulting English outcome post-its that would be taken forward by the team. The information selection contained a mixture of notes from the translation, contextual information and further creative elaborations. When counted by episode, this led to a dependent variable that was a cumulative count of the number of outcome post-its deriving from each episode.

\subsection{Coding Procedure and Inter-Coder Reliability Checks}

The dataset was coded by two independent student coders who were unaware of the research hypotheses. Each student coded the dataset in four iterations. One coder carried out all post-it categorization, cognitive referral numbering and coding for cognitive referrals, cognitive referral episodes, immediate creative elaboration and information selection. The other student coded for epistemic uncertainty and subsequent attentive returns. The first coder had assisted in the 
transcription and turn-taking segmentation of the sessions, and was therefore familiar with the content of the cognitive referents and the overall data.

Inter-coder reliability checks were conducted by asking a third coder to independently re-code $10 \%$ of the data, with reliability being estimated using Cohen's Kappa. All Kappa coefficients displayed fair-to-good or excellent inter-coder agreement (epistemic uncertainty $=.79$; immediate creative elaboration $=.83$; subsequent attentive returns $=.75$; information selection $=.58$ )

\section{RESULTS}

\subsection{Descriptive Findings}

A total of 173 Chinese post-its formed the basis for the design team's translation, elaboration and generation activities. Eighty-nine unique cognitive referral episodes were identified, constituting $64 \%$ of the data segments. These unique episodes were the basic unit of analysis, ranging in length from 1-49 segments $(\mathrm{M}=8.4, \mathrm{SD}=7.4)$. They contained an average of 0.46 epistemic uncertainty phrases per segment $(\mathrm{SD}=0.48$, Range $=0-2)$. Overall, $26 \%$ of all segments contained uncertainty phrases, which is a high percentage compared to past research, with around $15 \%$ of segments containing epistemic uncertainty being more typical (Ball \& Christensen, 2009; Wiltschnig et al., 2013). Such elevated levels of epistemic uncertainty perhaps derive from the inherent ambiguities associated with the translational and cross-cultural aspects of the present design situation. Segments arising within cognitive referral episodes contained uncertainty hedge words more frequently than segments arising outside of cognitive referral episodes $\left(\chi^{2}(1)=9.19, p=.002\right)$. However, uncertainty hedge words did not differ between segments arising within cognitive referral episodes and segments that immediately followed cognitive referral episodes $\left(\chi^{2}(1)=0.63\right.$, ns). Our analysis 
indicated that $35 \%$ of episodes were immediately followed (vs. not followed) by creative elaborative segments, and 55\% of the episodes had many (vs. few) subsequent attentive returns.

The design team made 85 notes relating to information selection, with 6 being clearly marked as 'categorical' post-its describing clusters of other post-its. The latter were excluded from the analysis, resulting in 79 post-its, 58 of which were coded as having been generated in reference to prior cognitive referral episodes. The post-it count by cognitive referral episode displayed a Poisson distribution, with 50,25, 11, 2, 0, 1 counts of 0-1-2-3-4-5 resulting post-its generated on their basis respectively (i.e., the majority of the notes were written with reference to a single episode). In 7 instances information was subsequently added to an existing post-it upon initial production. This adding of information mainly happened as a result of the later classification of the notes and was ignored for the present purposes.

\subsection{Mediation Analyses}

The binary codes for [M1] immediate creative elaboration and [M2] subsequent attentive returns were statistically unrelated $\left(\chi^{2}(1)=0.75, p=.39\right)$ illustrating independence of the hypothesized mediators, and further indicating that multicollinearity was not a concern in the subsequent regression models. To test the hypothesized relations we followed Baron and Kenny's (1986) stepbased procedure for testing mediation effects. It should be noted, however, that because of the different types of dependent variables in our analysis (binary for M1 and M2; Poisson distributed for Y), it was not possible to quantify the level of the direct effect versus the indirect mediation effect since distinct statistical tests were applied for testing individual relations (i.e., logistic regression for the relation between $[\mathrm{X}]$ epistemic uncertainty and the mediators $[\mathrm{a}, \mathrm{b}]$; GzLM Poisson regression for the relations between mediators $[a, b]$ and $[\mathrm{X}]$ epistemic uncertainty on $[\mathrm{Y}]$ 
information selection). As a result, the model test here should be considered as being primarily conceptual rather than a precise quantification of the direct and indirect effects. In all models we controlled for the video session the episodes derived from.

\section{Step1 $[X \rightarrow Y]$ Epistemic uncertainty and information selection}

A GzLM Poisson regression was run to test whether the level of epistemic uncertainty in the initial encounter with a cognitive referent predicted subsequent information selection. Overall, the model displayed acceptable goodness of fit $\left(\chi^{2} / \mathrm{df}=1.003\right)$. However, the analysis revealed that epistemic uncertainty did not predict information selection, although the odds ratio of 1.39 (95\% CI, 0.87 to $2.23), \mathrm{p}=.17$, was in the expected direction. This analysis suggests the absence of a direct effect of epistemic uncertainty on eventual information selection.

\section{Step2 [X $\rightarrow M 1]$ Epistemic uncertainty and immediate creative elaboration}

A logistic regression was conducted to test whether the level of epistemic uncertainty in a cognitive referral episode predicted immediate creative elaboration. Overall the model was significant $\left(\chi^{2}(3)\right.$ $=12.09, \mathrm{p}=.007$, Nagelkerke $\mathrm{R}^{2}=.18$ ), but epistemic uncertainty did not predict immediate creative elaboration $(p=.141)$, and with an odds ratio of 0.44 the results go in the opposite direction hypothesized (i.e., less uncertainty predicts immediate creative elaboration).

\section{Step2 [X $\rightarrow M 2]$ Epistemic uncertainty and subsequent attentive returns}

A logistic regression analysis was conducted to test whether the level of epistemic uncertainty in an episode predicted subsequent attentive returns. Overall the model was significant $\left(\chi^{2}(3)=12.05, \mathrm{p}\right.$ $=.007$, Nagelkerke $\mathrm{R}^{2}=.17$ ), with epistemic uncertainty significantly predicting subsequent attentive returns in the expected direction $(\mathrm{p}=.046)$, and with an odds ratio of 2.90 .

Step $3[M 1+X \rightarrow Y]$ Immediate creative elaboration and epistemic uncertainty onto information selection

A GzLM Poisson regression was run to predict information selection based on epistemic uncertainty in the initial encounter with the cognitive referent and immediate creative elaboration. 
Overall the model displayed acceptable goodness of fit $\left(\chi^{2} / \mathrm{df}=0.88\right)$. Immediate creative elaboration predicted information selection, odds ratio of 0.52 (95\% CI, 0.30 to $0.90, \mathrm{p}=.019)$, in the expected direction. Epistemic uncertainty approached, but did not reach, significance, odds ratio $1.61(95 \%$ CI, 0.97 to $2.67, \mathrm{p}=.067)$.

\section{Step $3[M 2+X \rightarrow Y]$ Subsequent attentive returns and epistemic uncertainty onto information selection}

A GzLM Poisson regression was run to predict information selection based on epistemic uncertainty in the initial encounter with the cognitive referent and subsequent attentive returns. Overall the model displayed acceptable goodness of fit $\left(\chi^{2} / \mathrm{df}=0.93\right)$. Subsequent attentive returns predicted information selection, odds ratio of $0.48(95 \% \mathrm{CI}, 0.27$ to $0.83, \mathrm{p}=.009)$, in the expected direction. Epistemic uncertainty did not reach significance, odds ratio 1.19 (95\% CI, 0.73 to 1.97, p $=.485)$

\section{Step $3[M 1+M 2+X \rightarrow Y]$ Both mediators and epistemic uncertainty onto information selection}

A GzLM model combining both mediators and epistemic uncertainty further illustrated that only subsequent attentive returns significantly predicted information selection (odds ratio: 0.54; 95\% CI, 0.30 to $0.97, \mathrm{p}=.038$ ), while both immediate creative elaboration (odds ratio: $0.62 ; 95 \% \mathrm{CI}, 0.35$ to $1.10, \mathrm{p}=.100$ ) and epistemic uncertainty (odds ratio: $1.36 ; 95 \% \mathrm{CI}, 0.80$ to $2.31, \mathrm{p}=.262$ ) were insignificant.

\subsection{Interpreting the Statistical Model}

Interpreting these results requires caution, since it was not possible to test for mediation using the same statistical test throughout given the Poisson-distributed outcome variable. As such, we were unable to calculate the direct versus indirect effects, but instead rely on interpreting the overall 
relations between the variables based on individual test results. Nonetheless, with appropriate caution the illustrated relations can best be described as approximating an 'opposing mediation', whereby the independent variable holds opposing relations to two distinct mediators that subsequently both positively affect the dependent variable (Figure 2). In opposing mediation the independent variable does not predict the dependent variable directly since the two mediators operate in opposite directions. In other words, high epistemic uncertainty triggers subsequent returns to the cognitive referent, while immediate creative elaboration is associated with epistemic certainty (although not reaching significance, and against the hypothesized direction based on past research). Both mediators positively affected information selection, with subsequent attentive returns proving to be the stronger predictor. Notably, epistemic uncertainty in itself does not significantly predict eventual information selection. In order to understand these patterns of effects in the dataset we present below extended, illustrative examples of the two 'routes' from epistemic uncertainty to information selection.

Insert Figure 2 about here

Figure 2. The suggested opposing mediation model arising from the analyses

\subsection{Qualitative Examples of the Two Routes from Epistemic Uncertainty to Information Selection}

\subsubsection{Example of high uncertainty leading to subsequent attentive returns and information selection}


Table 1 exemplifies how an episode with high uncertainty leads to subsequent attentive returns across episodes and ultimately to information selection. We enter the dialogue during a discussion about features for wearable devices connected to online services (Figure 3). In this fragment the post-its denoting 'Personal aspect', 'Human', and '2-way interaction' (information selection; Figure 4) are produced in response to Episode 40, which is related to the cognitive referent 'Interaction'.

The dialogue begins with Rose recalling an observation from the co-creation session. Rose refers, with high uncertainty ('I think' and 'kind of') to the analogy 'the car as a boyfriend' to explain product features like interaction and talking back to the user. Nina supplements Rose's comments with her own observation that 'it [the car/product] needs to be able to talk to you'. Rose confirms what Nina states in a way that links the 'talk' feature to the post-it 'Interaction' by saying 'Yeah, that kind of interaction' and using an air-quote gesture while saying the word 'interaction', implying that the word (or idea) belongs to the lead-users (Stivers \& Sidnell, 2005). Abby sums up what Rose said and writes 'Personal aspect' on a post-it. Kenny proposes with high uncertainty ('I think', 'it might', 'it could', 'maybe') that preferences might differ from person-to-person, which is confirmed by Tiffany ('mhh') and Abby ('yeah' followed by 'exactly'). Kenny repeats the word 'interaction', while drawing attention to the post-it and gesturing towards himself 'this has a relation to me'. Abby adds the text 'Different kinds of interaction' to the note. The episode is characterised by a high degree of uncertainty when Rose and Kenny talk, followed by repetitions and confirming utterances, which provides a display of understanding by the others.

In the next episode (Episode 41), Abby completes the post-it note. Nina refers to a statement by one of the lead users and again Rose validates her observations; Rose points at a Chinese post-it with the English text 'Alarm' two times using air-quote gestures while saying 'Alarm'. She explains the 'interaction feature' of the product using the Chinese post-it note 'Alarm' as a reference point, several times looking and pointing while she elaborates on what the lead-users meant during the co- 
creation session. Abby condenses the dialogue about product features (the alarm and push notifications) to 'Talk' as a finalizing remark and places the post-it next to another that also contains information on product features and services.

Insert Figure 3 about here

Figure 3. Setting (Video 15 at 39:14)
Insert Figure 4 about here

Figure 4. Post-it notes based on Episode 40

Rose and Tiffany then open a new episode (Episode 42) by referencing a new Chinese post-it 'Home doctor', and continue to talk about this service feature, while Abby writes another post-it with the word 'Human' (Figure 4), linking back to Episode 40. At the end of Table 1 Kenny writes '2-way interaction' on a post-it, which he sticks onto the bottom of the post-it that Abby previously wrote with the text 'Different kind of interaction'. Kenny's note can be linked to the topic of the product's interaction features from Episode 40, but its content also links to the post-it notes Abby placed on the wall: a 'Human' or 'Personal aspect' of a service, meaning a two-way interaction.

Overall, this example illustrates how uncertain dialogue is followed by subsequent attentive returns across episodes to the cognitive referent 'interaction', resulting finally in three new information selection post-its being produced. 
Table 1. Transcript Extract (Video 15, 227-238, run time 37:58)

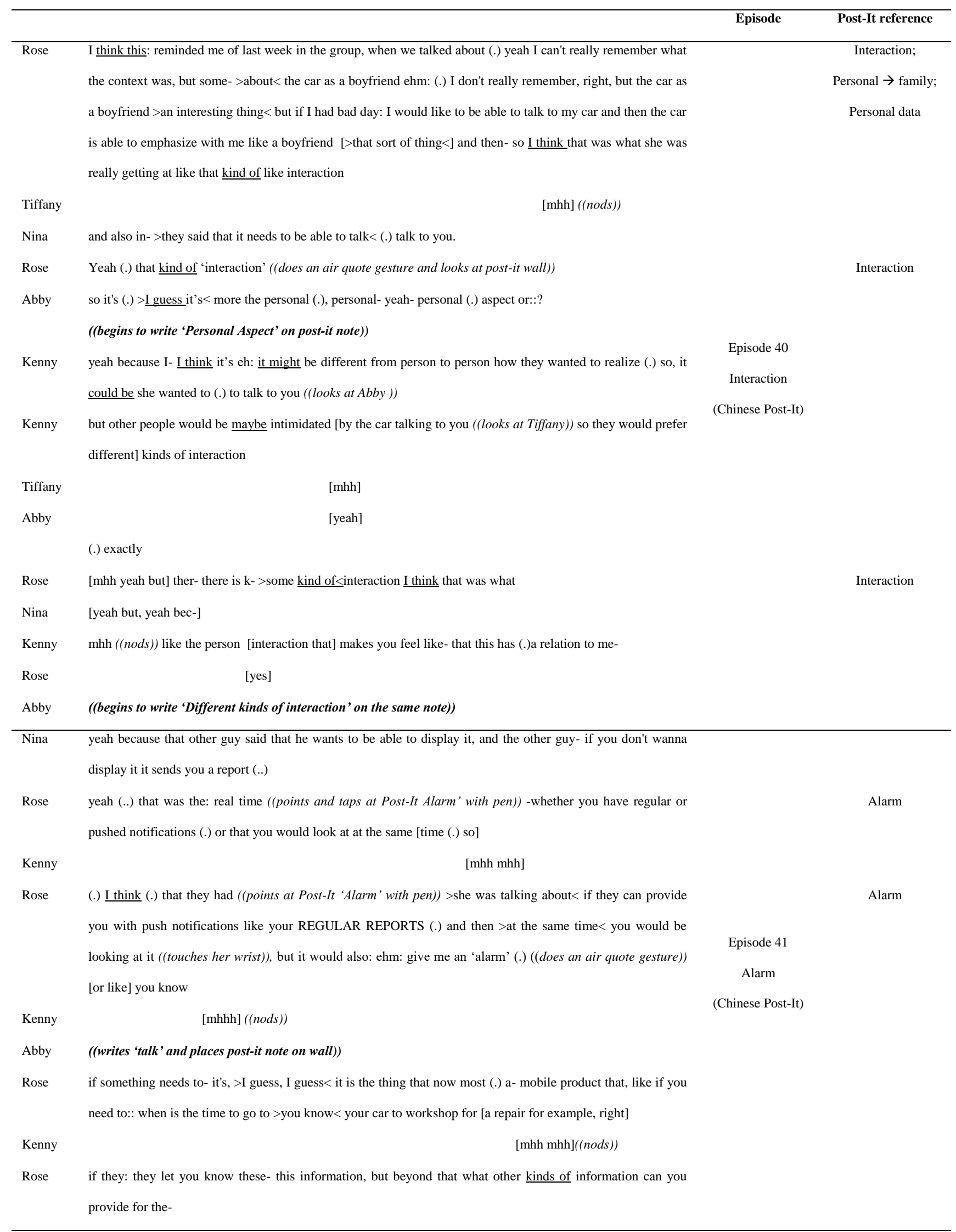




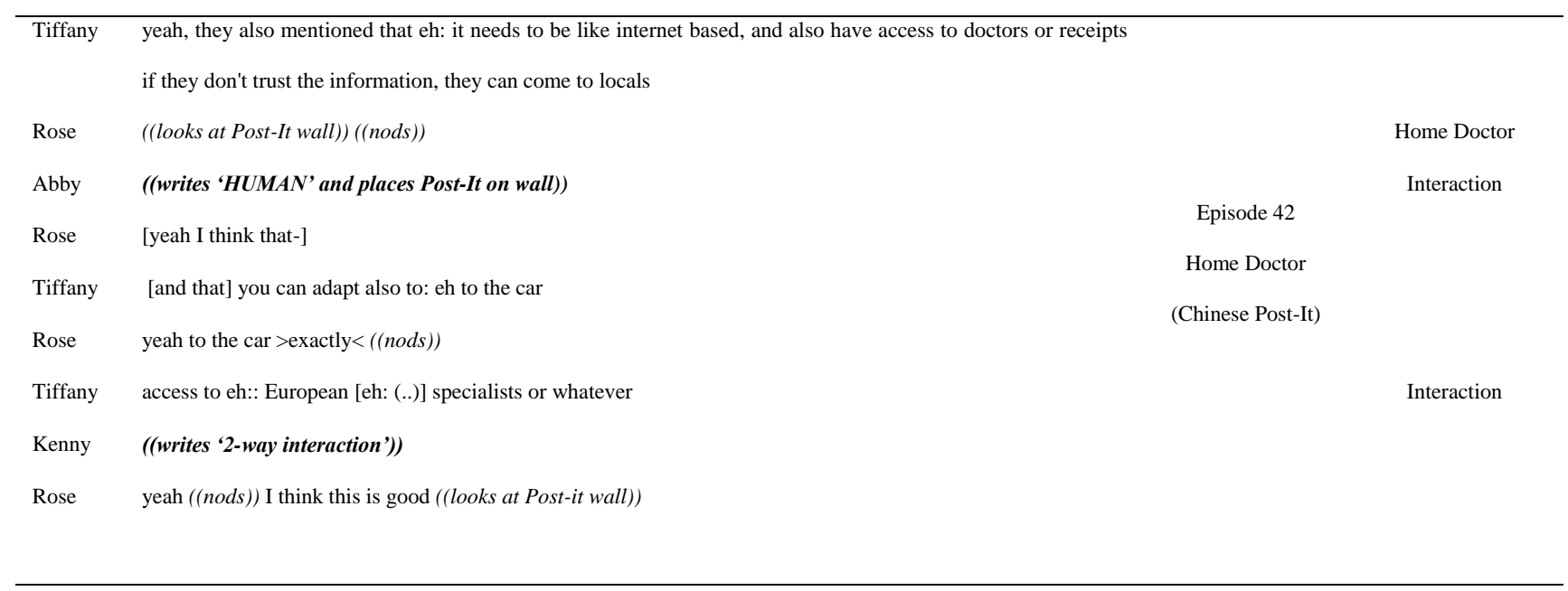

Note: Extract showing how low uncertainty triggers immediate cognitive elaboration and information selection.

Epistemic uncertainty hedge words are underlined and information extraction is in boldface.

3.3.2 Example of high certainty leading to immediate creative elaboration and information selection

This example is taken 3 minutes after the previous example. Episode 44 begins with Abby uttering an observation relating to the earlier co-creation session and the fact that the lead-users mentioned a 'Life time companion'. While Abby is talking and gesturing towards the Chinese post-it notes Rose is confirming her observations by pointing at the Chinese post-it with the translation 'Sustainable, lifelong'. Abby utters that 'this is exactly what we need to create', which both Tiffany and Rose agree with. Rose repeats Abby's statement 'exactly' in a manner that expresses no uncertainty (unlike the uncertainty hedge words in the previous episodes). Tiffany then goes on to confirm Abby's statement, adding that someone else also talked about this. The approving utterances illustrate that Abby's statement has solid grounding in the group. Rose finalizes the mutual decision; she asks Abby to write the point down. 
Tiffany continues to share her observations of what the lead-users mentioned, while Abby is writing 'Life time companion' on the post-it (Figure 5). Abby completes the post-it and fixes it to the wall, illustrating a shared representation. The group expresses excitement and certainty about the 'Life time companion' concept. Abby has 'ideas popping out' and Kenny finds it to be 'an awesome concept'. Abby says that 'it's so obvious' and 'of course', also expressing certainty on the matter. Tiffany adds that it is in fact something that 'they' (i.e. THE COMPANY) do already, which, even though the idea is not new, validates the concept further. In the last part of Episode 44 Rose begins to elaborate on the 'Life time companion' concept. Abby adds another post-it at the end of the episode, further elaborating on the idea ('Get attached to the service, -> continue to buy (COMPANY), the product').

The example illustrates how agreement and certainty within the dialogue are followed by immediate creative elaboration in the same episode, resulting finally in two new information selection post-its being produced.

Insert Figure 5 about here

Figure 5. Post-it note 
Table 2. Transcript Extract (Video 15, 262-268, run time 42:49)

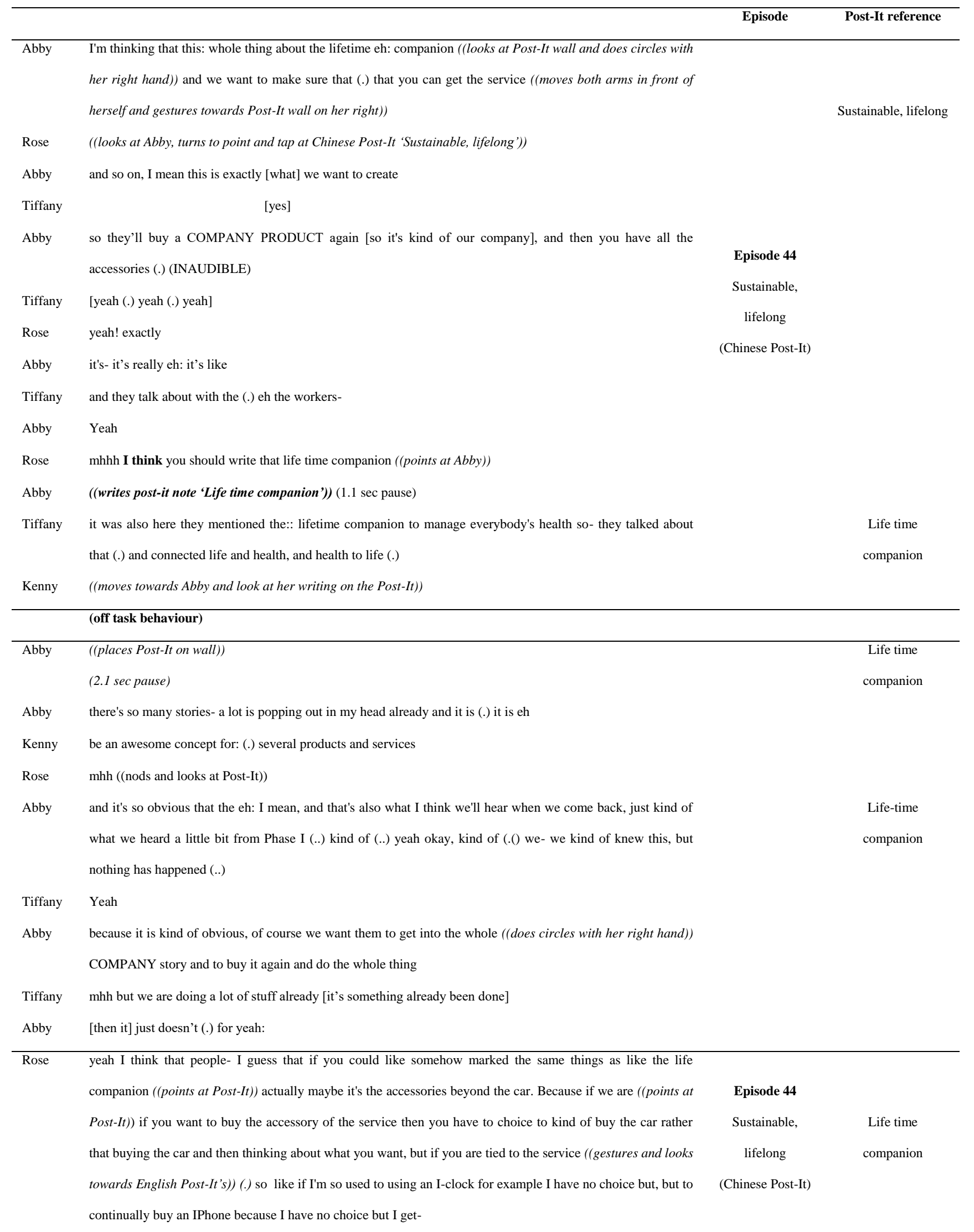




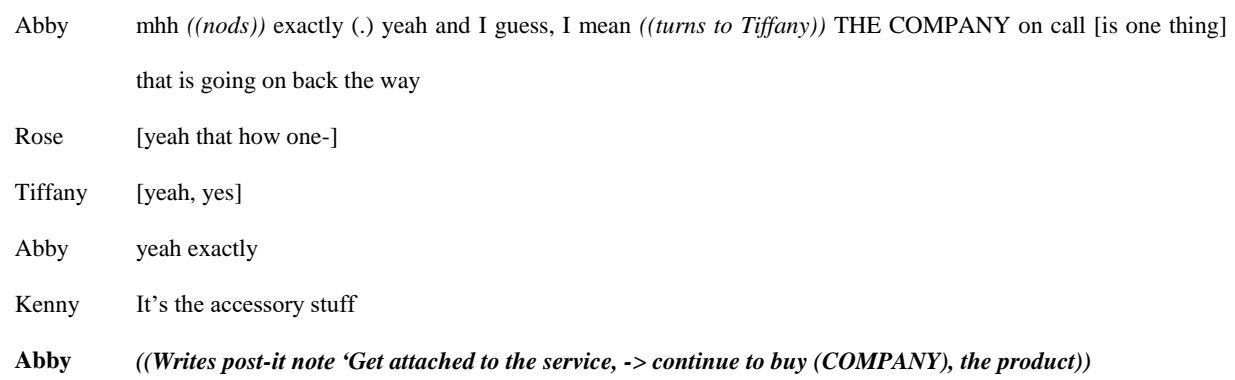

Note: Extract showing how low uncertainty triggers immediate cognitive elaboration and information selection. Epistemic uncertainty hedge words are underlined and information extraction is in boldface.

\section{DISCUSSION}

Previous research on epistemic uncertainty in design has focused on how such uncertainty triggers localized, creative reasoning such as analogizing and mental simulation aimed at uncertainty reduction (Ball \& Christensen, 2009; Ball, et al., 2010; Christensen \& Schunn, 2007, 2009; Wiltschnig et al., 2013). To date, however, no research has examined how epistemic uncertainty may also affect design behaviors beyond the localized micro-episode in which it is experienced, although phenomena such as the Zeigarnik effect (Zeigarnik, 1927), where people's memory for unsolved problems exceeds that for solved problems, suggests that designers may be alert to unresolved issues. The temporally-extended nature of the DTRS11 dataset afforded a unique opportunity to address this gap in existing knowledge through a direct test of the impact of epistemic uncertainty on creative design cognition both within and across episodes.

In analyzing the dataset for evidence of an association between epistemic uncertainty and creative design cognition we decided not to focus on analogizing and mental simulation, since these strategies were not particularly in evidence. Instead, we examined how the level of epistemic uncertainty associated with a cognitive referent predicts the repeated referral back to that referent in 
subsequent dialogue. In the present dataset such cognitive referents took the form of the post-it notes deriving from Chinese lead-users. Our analysis was driven by the dual hypotheses that increased uncertainty associated with a cognitive referent would engender: (1) immediate creative elaboration of that referent aimed at resolving uncertainty and determining information selection; and (2) subsequent attentive returns to that cognitive referent aimed at resolving lingering uncertainty and again determining information selection. In sum, we proposed a 'double mediation model' (Figure 1) in which epistemic uncertainty on the initial encounter with an individual post-it predicts both the immediate occurrence of local, creative micro-episodes that mediate the formation of new post-its (information selection) as well as the occurrence of subsequent attentive returns across episodes that also mediate the formation of new post-its (further information selection).

Our results challenge some aspects of our a priori assumption that the uncertainty associated with cognitive referents (post-its) would predict localized, creative elaboration and subsequent attentive returns, with both types of creative activities mediating eventual information selection. First, it was apparent that increased epistemic uncertainty in relation to cognitive referents did not immediately trigger creative cognitive events. Instead, it was epistemic certainty in relation to cognitive referents that engendered immediate, creative reasoning and information elaboration. In accounting for this opposite-to-predicted effect we speculate that uncertainty might have been so highly elevated in the present design context as almost to force the design team to opt strategically to make the most of any certain information at hand, immediately latching onto this and utilizing it as a way to establish a stable framework for subsequent work. Achieving a stable framework might be especially critical in design tasks that involve having to respond to end-user knowledge, where it is important to commence with some certainties as a foundation to build upon. In this way it is possible that epistemic uncertainty triggers a 'metacognitive switch' that is highly bounded by the prevailing context. If epistemic uncertainly is felt to be uniformly high then designers may opt to work 
creatively in the immediate term with information that feels more certain, whereas if uncertainty shows greater fluctuation then designers may opt to expend more immediate effort on resolving uncertain aspects of the design.

The idea of epistemic uncertainty driving a metacognitive switch mechanism is gaining credibility in the literature on human reasoning (e.g., Ackerman \& Thompson, 2014; Ball \& Stupple, 2016; Thompson et al., 2011; Thompson et al., 2013). Interestingly, too, it is becoming increasingly clear that the strategic decisions people make on the basis of metacognitive experiences are often determined by relative rather than absolute perceptions relating to perceived uncertainty (Wänke \& Hansen, 2015). In other words, it appears that dynamic shifts from perceived certainty to perceived uncertainty are critical for determining strategic decisions about the kind of reasoning required at any particular point in time. These important conceptual ideas align well with our view that in some design contexts epistemic uncertainty may fluctuate extensively, with bouts of uncertainty leading to immediate, creative processing, whereas in other design contexts epistemic uncertainty may provide a more global and stable backdrop to ongoing activity, potentially leading to isolated moments of certainty triggering immediate creative processing, as observed here. It is noteworthy that in a post-study interview the leader of the design team spoke of his perception of post-its as 'emotional triggers', evidencing an understanding that the emotional qualities of post-its could be a driver for subsequent design processes, although he did not specifically focus on epistemic uncertainty.

A second key finding is that we have shown for the first time how epistemic uncertainty can promote subsequent attentive returns to a cognitive referent within the design process, since our analyses demonstrated a predicted correlation between initial, epistemic uncertainty that was linked to cognitive referents and such subsequent attentive returns. This finding corroborates our underpinning proposal that epistemic uncertainty may affect design behavior that extends beyond 
the localized micro-situation in which the uncertainty is experienced. The idea that epistemic uncertainty can have far-reaching consequences seems novel and important and would be worth exploring further.

A third finding is that while epistemic uncertainty did not directly predict the information that was eventually selected, both immediate creative elaboration and subsequent attentive returns did predict information selection, with subsequent attentive returns being the stronger predictor. The fact that both mediator variables were predictive of information selection is interesting, although not surprising. In essence, this finding indicates that once designers have engaged in creative development activity in relation to a cognitive referent this activity then forms the basis of information selection for down-stream design work. Arguably, too, it might be expected that subsequent attentive returns would emerge as the stronger predictor of information selection compared to immediate creative elaboration given that the designers have presumably returned to unresolved issues because of their perceived importance for design success (i.e., the Zeigarnik effect may not only impact on memory for unresolved issues but may also impact information selection for subsequent processing).

Before concluding, we note some caveats in relation to our findings, which primarily center on statistical issues. First, the small sample-size associated with our analysis will have reduced the reliability of our results. Second, we recognize the relatively small effect sizes arising from our analyses. Third, we reiterate the interpretative problems arising from our inability to test our proposed mediation model using the same regression methods because of the particular statistical properties of the variables underpinning the model. Our approach was to undertake a conceptual mediation analysis, although this didn't allow us to formalize decisively the evidence for direct and mediated effects between predictor variables in relation to the dependent variable of information selection. Our interpretation of findings therefore needs to be treated with an appropriate degree of 
caution. That said, we contend that our approach and observations hold promise for identifying more pervasive and enduring impacts of epistemic uncertainty on creative design cognition that are potentially mediated through salient cognitive referents. In addition, our research contributes to a growing appreciation of uncertainty as a salient aspect of design that determines the dynamics of ongoing creative design reasoning and decision making (e.g., Stempfle \& Badke-Schaub, 2002; Tracey \& Hutchinson, 2016).

\section{REFERENCES}

Ackerman, R., \& Thompson, V. A. 2014. “Meta-reasoning: What can we learn from meta-memory”. In: A. Feeney, \& V. A. Thompson (eds.) Reasoning as memory. Hove, UK: Psychology Press.

Alcaide-Marzal, J., Diego-Más, J. A., Asensio-Cuesta, S., \& Piqueras-Fiszman, B. 2013. “An exploratory study on the use of digital sculpting in conceptual product design”. Design Studies, 34(2), 264-284.

Alter, A. L., \& Oppenheimer, D. M. 2009. "Uniting the tribes of fluency to form a metacognitive nation”. Personality \& Social Psychology Review, 13(3), 219-235.

Alter, A. L., Oppenheimer, D. M., Epley, N., \& Eyre, R. N. 2007. ”Overcoming intuition: Metacognitive difficulty activates analytic reasoning”. Journal of Experimental Psychology: General, 136(4), 569-576.

Baron, R. M., \& Kenny, D. A. 1986. “The moderator-mediator variable distinction in social psychological research: Conceptual, strategic and statistical considerations". Journal of Personality and Social Psychology, 51(6), 1173-1182.

Ball, L. J., \& Christensen, B. T. 2009. “Analogical reasoning and mental simulation in design: Two strategies linked to uncertainty resolution”. Design Studies, 30(2), 169-186.

Ball, L. J., Onarheim, B., \& Christensen, B. T. 2010. "Design requirements, epistemic uncertainty and solution development strategies in software design”. Design Studies, 31(6), 567-589.

Ball, L. J., \& Stupple, E. J. N. 2016. "Dual reasoning processes and the resolution of uncertainty: The case of belief bias”. In: L. Macchi, M. Bagassi, \& R. Viale (eds.) Cognitive unconscious and human rationality. Cambridge, MA: MIT Press. pp. 143-165 
Beheshti, R. 1993. “Design decisions and uncertainty”. Design Studies, 14(1), 85-95.

Chan, J., Paletz, S. B., \& Schunn, C. D. 2012. “Analogy as a strategy for supporting complex problem solving under uncertainty". Memory \& Cognition, 40(8), 1352-1365.

Christensen, B. T., Ball, L. J. \& Halskov, K. (eds.). 2017. “Analysing Design Thinking: Studies of Cross-Cultural CoCreation”. Leiden: CRC Press/Taylor \& Francis.

Christensen, B. T. \& Abildgaard, S. J. J. 2017. "Inside the DTRS11 Dataset: Background, Content, and Methodological Choices”. In: Christensen, B. T., Ball, L. J. \& Halskov, K. (eds.) Analysing Design Thinking: Studies of CrossCultural Co-Creation. Leiden: CRC Press/Taylor \& Francis.

Christensen, B. T., \& Ball, L. J. 2014. "Studying design cognition in the real world using the 'In Vivo' methodology". In: P. Rodgers \& J. Yee (eds.) The Routledge Companion to Design Research Abingdon, UK: Routledge. pp. 317-328.

Christensen, B. T., \& Schunn, C. D. 2005. "Spontaneous access and analogical incubation effects". Creativity Research Journal, 17(2-3), 207-220.

Christensen, B. T., \& Schunn, C. D. 2007. "The relationship of analogical distance to analogical function and preinventive structure: The case of engineering design”. Memory \& Cognition 35(1), 29-38.

Christensen, B. T., \& Schunn, C. D. 2009. "The role and impact of mental simulation in design". Applied Cognitive Psychology, 23(3), 327-344.

Dorst, K., \& Cross, N. 2001. "Creativity in the design process: Co-evolution of problem-solution”. Design Studies, $22(5), 425-437$.

Dove, G., Abildgaard, S. J., Biskjaer, M. M., Hansen, N. B., Christensen, B. T. \& Halskov, K. 2017 “Grouping Notes Through Nodes: The Functions of Post-It ${ }^{\mathrm{TM}}$ Notes in Design Team Cognition”. In: Christensen, B. T., Ball, L. J. \& Halskov, K. (eds.) Analysing Design Thinking: Studies of Cross-Cultural Co-Creation. Leiden: CRC Press/ Taylor \& Francis.

D’souza, N. \& Dastmalchi, M. 2017 “Comfy” Cars for the “Awesomely Humble”: Exploring Slangs and Jargons in a Cross-Cultural Design Process". In: Christensen, B. T., Ball, L. J. \& Halskov, K. (eds.) Analysing Design Thinking: Studies of Cross-Cultural Co-Creation. Leiden: CRC Press/ Taylor \& Francis. 
Dunbar, K. 1995. “How scientists really reason: Scientific reasoning in real-world laboratories”. In: R. J. Sternberg and J. E. Davidson (eds.) The nature of insight. Cambridge, MA: MIT Press. pp. 365-395

Dunbar, K. 1997. "How scientists think: On-line creativity and conceptual change in science”. In: T. Ward, S. M. Smith \& J. Vaid (eds.) Creative thought: An investigation of conceptual structures and processes). Washington DC: American Psychological Association. pp. 461-493

Ericsson, K. A., \& Simon, H. A. 1999. “Protocol analysis: Verbal reports as data”. Cambridge, MA: MIT Press.

Finke, R. A., Ward, T. B., \& Smith, S. M. 1992. “Creative cognition: Theory, research, and applications”. Cambridge, MA: MIT Press.

Gilhooly, K. J. in press. "Incubation, problem solving and creativity". In: L. J. Ball \& V. A. Thompson (eds.) The Routledge international handbook of thinking and reasoning. Oxford, UK: Routledge.

Houghton, D. P. 1998. "Historical analogies and the cognitive dimension of domestic policymaking". Political Psychology, 19(2), 279-303.

Howard, T. J., Culley, S. J., \& Dekoninck, E. 2008. "Describing the creative design process by the integration of engineering design and cognitive psychology literature”. Design Studies, 29(2), 160-180.

McDonnell, J. 2015. "Gifts to the future: Design reasoning, design research, and critical design practitioners". She Ji: The Journal of Design, Economics, and Innovation, 1(2), 107-117.

Nersessian, N. J. 2009. "How do engineering scientists think? Model-based simulation in biomedical engineering research laboratories". Topics in Cognitive Science, 1(4), 730-757.

Paletz, S. B. F., Sumer, A. \& Miron-Spektor, E. 2017 "Psychological Factors Surrounding Disagreement in Multicultural Design Team Meetings”. In: Christensen, B. T., Ball, L. J. \& Halskov, K. (eds.) Analysing Design Thinking: Studies of Cross-Cultural Co-Creation. Leiden: CRC Press/ Taylor \& Francis.

Schön, D. A. 1983. “The reflective practitioner: How professionals think in action”. New York: Basic Books.

Schön, D. A., \& Wiggins, G. 1992. “Kinds of seeing and their functions in designing”. Design Studies, 13(2), 135-156.

Seifert, C. M., Meyer, D. E., Davidson, N., Patalano, A. L., \& Yaniv, I. 1995. D”emystification of cognitive insight: Opportunistic assimilation and the prepared-mind perspective”. In: R. J. Sternberg \& J. E. Davidson (eds.) The nature of insight. Cambridge, MA: MIT Press. Pp65-124. 
Sio, U. N., \& Ormerod, T. C. 2009. "Does incubation enhance problem solving? A meta-analytic review". Psychological Bulletin, 135(1), 94-120.

Stempfle, J., \& Badke-Schaub, P. 2002. "Thinking in design teams-an analysis of team communication". Design Studies, 23(5), 473-496.

Stivers, T., \& Sidnell, J. 2005. “Introduction: Multimodal interaction”. Semiotica, 2005 (156), 1-20.

Thompson, V. A., Prowse Turner, J., \& Pennycook, G. 2011. "Intuition, reason, and metacognition". Cognitive Psychology, 63(3), 107-140.

Thompson, V. A., Prowse-Turner, J., Pennycook, G. R., Ball, L. J., Brack, H. M., Ophir, Y., \& Ackerman, R. 2013. "The role of answer fluency and perceptual fluency as metacognitive cues for initiating analytic thinking". Cognition, 128(2), 237-251.

Tracey, M. W., \& Hutchinson, A. 2016. "Uncertainty, reflection, and designer identity development”. Design Studies, 42, 86-109.

Trickett, S. B., Trafton, J. G., Saner, L. D., \& Schunn, C. D. 2005. "I don't know what's going on there: The use of spatial transformations to deal with and resolve uncertainty in complex visualizations”. In: M. Lovett \& P. Shah (eds.) Thinking with data. Mahwah, NJ: Lawrence Erlbaum Associates, Inc. pp. 65-86.

Ülkümen, G., Fox, C. R., \& Malle, B. F. 2016. "Two dimensions of subjective uncertainty: Clues from natural language". Journal of Experimental Psychology: General, 145(10), 1280-1297.

Wänke, M., \& Hansen, J. 2015. "Relative processing fluency”. Current Directions in Psychological Science, 24(3), 195-199.

Wiltschnig, S., Christensen, B. T., \& Ball, L. J. 2013. "Collaborative problem-solution co-evolution in creative design”. Design Studies, 34(5), 515-542.

Yaniv, I. \& Meyer, D. E. 1987. ”Activation and metacognition of inaccessible stored information: Potential bases for incubation effects in problem solving". Journal of Experimental Psychology: Learning, Memory, and Cognition, 13, 187-205.

Zeigarnik, B. 1927. "Das behalten erledigter und unerledigter handlungen”. Psychologische Forschung, 9, 1-85. 